\title{
Quantitative OCT-Based Longitudinal Evaluation of Intracorneal Ring Segment Implantation in Keratoconus
}

\author{
Pablo Pérez-Merino, ${ }^{1}$ Sergio Ortiz, ${ }^{1}$ Nicolas Alejandre, ${ }^{1,2}$ Ignacio Jiménez-Alfaro, ${ }^{1,2}$ \\ and Susana Marcos ${ }^{1}$ \\ ${ }^{1}$ Instituto de Óptica "Daza de Valdés," Consejo Superior de Investigaciones Científicas (CSIC), Madrid, Spain \\ ${ }^{2}$ Fundación Jiménez Díaz, Madrid, Spain
}

Correspondence: Pablo Pérez-Merino, Instituto de Óptica "Daza de Valdés," Consejo Superior de Investigaciones Científicas, C/ Serrano, 121, 28006 Madrid, Spain; p.perez@io.cfmac.csic.es.

Submitted: May 13, 2013

Accepted: July 24, 2013

Citation: Pérez-Merino P, Ortiz S, Alejandre N, Jiménez-Alfaro I, Marcos S. Quantitative OCT-based longitudinal evaluation of intracorneal ring segmentation implantation in karatoconus. Invest Ophthalmol Vis Sci. 2013;54:6040-6051. DOI:10.1167/ iovs.13-12401
Purpose. To characterize the geometrical properties of keratoconic corneas upon intracorneal ring segments (ICRS) implantation, using custom-developed optical coherence tomography (OCT).

Methods. Ten keratoconic corneas were measured pre- and post-ICRS surgery (7, 30, and 90 days). Corneal topographic and pachymetric maps were obtained from three-dimensional (3D) images acquired with OCT, provided with custom algorithms for image analysis, distortion correction, and quantification. The 3D positioning of the ICRS was also estimated longitudinally, relative to the pupil center and iris plane.

Results. Preoperatively, the average corneal radii of curvature were $7.02 \pm 0.54 \mathrm{~mm}$ (anterior) and $5.40 \pm 0.77 \mathrm{~mm}$ (posterior), and the minimum corneal thickness was $384 \pm$ $60 \mu \mathrm{m}$. At 90 days, the average corneal radii of curvature were $7.26 \pm 0.53 \mathrm{~mm}$ (anterior) and $5.44 \pm 0.71 \mathrm{~mm}$ (posterior), and the minimum corneal thickness was $396 \pm 46 \mu \mathrm{m}$. ICRS implantation produced a significant decrease of corneal power (by $1.71 \pm 1.83$ diopters [D] at 90 days). Corneal irregularities (defined by high order Zernike terms of the corneal elevation maps) and the corneal thickness distribution decreased in some patients and increased in others. The 3D ICRS depth matched the planned ICRS depth well (within 23.93 $\pm 23.49 \mu \mathrm{m}$ ). On average, ICRS showed an overall tilt of $-6.8 \pm 2.6^{\circ}$ (temporal) and $-2.1 \pm$ $0.8^{\circ}$ (superior) at 7 days.

ConcLusions. Spectral OCT (sOCT) provided with distortion correction and analysis tools, is an excellent instrument for evaluating the changes produced by ICRS in keratoconic corneas, and for analyzing the 3D ICRS position during the follow up. ICRS produced flattening on the anterior corneal surface, although the benefit for corneal surface regularization varied across patients.

Keywords: optical coherence tomography (OCT), keratoconus, intracorneal ring segments (ICRS), corneal topography
$\mathrm{K}$ eratoconus is a corneal disorder that affects the shape and structure of the cornea, seriously compromising visual performance. The distorted corneal geometry severely reduces the optical quality of the eye, making difficult its correction with spectacles or contact lenses. Intracorneal ring segments (ICRS) are an increasingly used surgical alternative to delay corneal transplant and improve visual quality in keratoconus. ${ }^{1-6}$ ICRS are expected to improve the optical quality of the cornea by reducing local steepening and increasing corneal symmetry. ${ }^{3-6}$ Several studies have reported anterior and posterior corneal geometry in keratoconus and its change upon ICRS implantation. Assessment of ICRS implantation has been performed using different imaging techniques including slitscanning corneal topography (Orbscan; Orbtek, Inc., Salt Lake City, UT), ${ }^{7}$ Scheimpflug imaging (Pentacam; Oculus, Inc., Lynnwood, WA $)^{8}$ or ultrasound biomicroscopy.9,10 Some inherent limitations of these techniques include optical and geometrical distortion in the acquired images, low resolution, or acquisition times exceeding typical eye motions. ${ }^{11-13}$ However, quantitative image-based techniques would be extremely helpful to evaluate objectively the keratoconic cornea and the performance of ICRS.

An excellent imaging alternative with improved acquisition time and resolution is optical coherence tomography (OCT), which provides high-speed noncontact three-dimensional (3D) imaging of the anterior segment of the eye with micron resolution. ${ }^{14,15}$ Several OCT studies have reported thickness, power, curvature, and topography in keratoconus, ${ }^{16-21}$ as well as the implantation depth of ICRS in keratoconic corneas. ${ }^{22-24}$ However, OCT is generally subject to distortions. Fan distortion arises from the scanning architecture of the system (two mirrors positioned in different planes and collimating lens) and is a result of a combination of geometrical aberrations, including field distortion, astigmatism, and spherical aberration. As a result of fan distortion a flat surface imaged through an uncorrected system appears curved, at least in one meridian. Optical distortion arises from refraction of surfaces imaged through refracting surfaces/media. Due to these distortions OCT images need to be corrected for quantification. ${ }^{25,26}$ Fan (following instrument calibration) and optical distortion (through preceding surfaces) are corrected using 3D ray tracing 
analysis. $^{25-28}$ We have previously reported on distortioncorrection methods and image analysis tools for quantification of OCT images, and demonstrated the accuracy of OCT-based corneal topography in healthy ${ }^{25,27,28}$ and irregular ${ }^{29}$ corneas. We have also presented dedicated tools to quantify threedimensionally the positioning of ICRS in treated corneas. ${ }^{29}$ In this study, we applied high-speed spectral domain OCT and the developed technology for fully characterizing the cornea in keratoconus and for evaluating longitudinally anterior and posterior corneal topographic changes following ICRS implantation, tracking also the position of the ICRS with time.

The study of the changes in anterior and posterior corneal topography with ICRS using demonstrated accurate methodology will give insights on the performance of the ICRS treatment, and the potential reasons behind the limited success of some of the procedures in some patients. In addition, the longitudinal characterization of the ICRS positioning in 3D will shed light into ongoing debates on the stability of ICRS, and on reported complications such as ICRS rotation or migration. ${ }^{30,31}$ This information will be also extremely valuable to understand the mechanism of action of ICRS and provide feedback to biomechanical models of the cornea and ICRS implants ${ }^{32}$ to increase the predictability of this treatment.

\section{Materials AND Methods}

\section{Patients}

Ferrara-like ICRS (FerraraRing; AJL Ophthalmics, Vitoria, Spain) were implanted in 10 corneas of 10 patients (ages ranging from 23-41 years) with diagnosed keratoconus. Manual and femtosecond laser-assisted techniques were performed for implanting ICRS. The Table summarizes the clinical profile of the patients and the specifications of the surgical procedure for ICRS implantation in each patient. Depending on the preoperative corneal topography and refraction, one or two segments were implanted, equidistant to the incision site. If the corneal coma axis and the flattest meridian differed less than $60^{\circ}$ the incision was performed in the steepest meridian and a $160^{\circ}$ segment was placed inferiorly (subject number [S\#] $3, \mathrm{~S} \# 9$ ). If anterior corneal astigmatism was higher than 5 diopters (D), an additional $90^{\circ}$ segment was inserted superiorly (S\#4, S\#6, S\#8, and $S \# 10$ ). If the spherical equivalent was higher than $9 \mathrm{D}$ a $120^{\circ}$ segment was instead inserted superiorly $(\mathrm{S} \# 1)$. If anterior corneal astigmatism was lower than $3 \mathrm{D}$, coma was higher than $2 \mu \mathrm{m}$ (for 5-mm diameter), the corneal coma axis and the flattest meridian differed more than $60^{\circ}$ and bestcorrected visual acuity (BCVA) was below 20/30, a $210^{\circ}$ segment was placed inferiorly with its center along the corneal coma axis ( $\mathrm{S} \# 5$ and $\mathrm{S} \# 7$ ). If the patient showed a regular myopic astigmatism higher than $4 \mathrm{D}$, the incision was performed in the steepest meridian, and two $120^{\circ}$ segments were inserted $(\mathrm{S} \# 2)$. All eyes were examined before and after ICRS implantation at 7, 30, and 90 days. The study was approved by institutional review boards at Fundación JiménezDíaz) and followed the tenets of the Declaration of Helsinki. The patients signed a consent form and they were aware of the nature of the study. Figure 1 shows the OCT en face image for each patient, illustrating the ICRS frontal position at 7 days after surgery.

\section{Custom Spectral Domain OCT System}

The OCT images were acquired using a custom developed spectral domain OCT system (from a collaborative effort with Copernicus University, Torun, Poland) previously described in detail in previous publications. ${ }^{15,25,28,29}$ Briefly, the setup is based on a fiber optics Michelson interferometer configuration with a superluminiscent diode $\left(\lambda_{0}=840 \mathrm{~nm}, \Delta \lambda=50 \mathrm{~nm}\right)$ as a light source and a spectrometer consisting of a volume diffraction grating, and a complementary metal-oxide-semiconductor (CMOS) camera as a detector. The effective acquisition speed is 25,000 A-Scans/s. The axial range is $7 \mathrm{~mm}$ in depth, resulting in a theoretic pixel resolution of $3.4 \mu \mathrm{m}$. The axial resolution is $6.9 \mu \mathrm{m}$.

Images were acquired while the patients fixated a Maltese cross fixation stimulus presented on a mini display implemented in a secondary channel of the OCT instrument. The images of the cornea were acquired centered at the specular reflection, which was used as a reference in the image acquisition throughout the different sessions. Sets of 3D images were captured approximately 5 seconds after blinking. At least five repeated measurements were collected in each condition, and processed independently. Data are given in terms of average and SDs. Images containing artifacts (i.e., eyelids), which precluded corneal surface analysis within the optical zone were excluded, for statistical analysis five measurements were used in this study.

Measurements were collected in a $10 \times 12-\mathrm{mm}$ area, using a horizontal raster scan. Each $3 \mathrm{D}$ data set consisted of a total of 50 B-scans composed by a collection of 360 A-scans. The total acquisition time of a $3 \mathrm{D}$ data set was 0.72 seconds.

\section{OCT Image Processing: Corneal Surface Analysis and ICRS Segmentation}

Custom algorithms to analyze the OCT 3D corneal images, and to correct for optical and fan distortion have been described previously. $25,27-29,33,34$ In addition, we have previously presented dedicated image processing tools for automatic analysis of ICRS from OCT images. Processing tools included denoising algorithms, automatic identification of 3D structures (cornea, iris, ICRS), multilayer segmentation, and distortion corrections. Figure 2 illustrates the image analysis in $S \# 2$ for pre and postoperative measurements $(7,30$, and 90 days).

The pupil center (obtained from the automatically identified iris volume) was used as a reference in the analysis of pre- and postoperative measurements. Corneal elevation maps were reported within the optical zone defined by the ICRS and the natural pupil. The center of the implanted ICRS was obtained from the automatically identified ICRS volume, and its shift from the pupil center estimated for registration of pre- and postoperative measurements. The optical zone is defined by the $4-\mathrm{mm}$ diameter circular zone inside the circumference defined by the ICRS radius, and centered at the pupil center. ${ }^{29}$

Corneal shape was described in terms of the radii of curvature and asphericities from fittings to a sphere and a biconicoid (anterior cornea: $R$ [sphere]; Rx and Ry, Qx and Qy [biconicoid]; posterior surface: $R$ [sphere]), and corneal elevation maps were also fitted to Zernike polynomial expansions (sixth order) using the center of the optical ICRS zone described in the previous paragraph as the reference. This way, the same optical zone was analyzed in the pre- and postoperative conditions. ${ }^{29}$

Topographic and thickness maps were displayed in a grid square of $100 \times 100$ points in the $4-\mathrm{mm}$ of diameter optical ICRS zone in order to ensure quantitative analysis of the optical zone without the ICRS influence. Topographic maps were represented as the difference of corneal elevation data from the reference sphere, in the so called "height representation," with warm colors representing points that are higher than the reference surface and cool colors representing points below the reference. Both anterior and posterior corneal surfaces were fitted by Zernike polynomial expansions (note that these are fits to surface elevations, not corneal wave aberrations). 
The symmetry of the corneal elevation maps was obtained using the Root Mean Square error (RMS) of the asymmetric terms of the corneal elevation Zernike expansion (RMS_asym). RMS_asym was therefore defined as the RMS for astigmatism, coma, trefoil, tetrafoil, pentafoil, and hexafoil terms $\left(\mathrm{Z}_{2}{ }^{-2}, \mathrm{Z}_{2}{ }^{2}\right.$, $\mathrm{Z}_{3}{ }^{-3}, \mathrm{Z}_{3}{ }^{-1}, \mathrm{Z}_{3}{ }^{1}, \mathrm{Z}_{3}{ }^{3}, \mathrm{Z}_{4}^{-4}, \mathrm{Z}_{4}^{-2}, \mathrm{Z}_{4}{ }^{2}, \mathrm{Z}_{4}^{4}, \mathrm{Z}_{5}{ }^{-5}, \mathrm{Z}_{5}{ }^{-3}, \mathrm{Z}_{5}^{-1}, \mathrm{Z}_{5}{ }^{1}$, $Z_{5}{ }^{3}, Z_{5}{ }^{-5}, Z_{6}{ }^{-6}, Z_{6}{ }^{-4}, Z_{6}{ }^{-2} Z_{6}{ }^{2}, Z_{6}{ }^{4}$, and $Z_{6}{ }^{6}$ ) of corneal height maps (not to be confused with wave aberration maps). RMS_asym was evaluated both including and excluding astigmatism coefficients.

Thickness maps were calculated from direct subtraction of the posterior corneal surface from the anterior corneal surface. RMS thickness maps were used to assess the regularity of the thickness distribution. RMS_thicknessmap is therefore defined as the deviation of the thickness maps from a uniform pachymetry across the cornea.

Corneal power (D) was calculated by using the paraxial formula with both the corneal (1.376) and the aqueous (1.336) refractive indices. All metrics were computed for a 4-mm optical ICRS zone.

3D ICRS positioning was described by the following parameters: 3D ICRS depth, defined as the distance between the center of mass of the ICRS and the anterior corneal surface, and ICRS tilt, defined as the angle between the ICRS axis (normal to the ICRS plane) and the pupillary axis (normal to the pupil plane). Positive tilts around $x$-axis indicate a forward tilt of the nasal part (OD)/temporal part (OS) of the ICRS plane with respect to the pupil plane. Positive tilts around $y$-axis indicate a forward shift of the inferior part of the ICRS plane with respect to the pupil plane. Figure 3 illustrates the definitions of the pupilary plane (and axis), ICRS plane (and axis), and ICRS tilt (Fig. 3a), as well as the tilt sign notations (Figs. 3b, 3c).

The changes in corneal geometry and ICRS position were analyzed statistically using an ANOVA (general linear model for repeated measurements). Significant levels (ANOVA and pairwise two-tailed comparison $t$-test) were set at $P$ less than 0.05 . The statistical tests were performed using SPSS software (SPSS, Inc., Chicago, IL).

\section{Results}

\section{Longitudinal Changes of Anterior Corneal Surface Geometry and Topography}

Radius of curvature and asphericity of the anterior corneal surface were obtained from sphere $(R)$ and biconicoid $(\mathrm{Rx}, \mathrm{Qx}$; Ry, Qy) fittings in a 4-mm optical zone.

Figure $4 \mathrm{a}$ shows averaged horizontal and vertical anterior corneal radii of curvature ( $\mathrm{Rx}$ and $\mathrm{Ry}$ ) in each eye before and at 7, 30, and 90 days after ICRS implantation. The intrasubject repeatability in the estimated anterior radius of curvature across repeated measurements is high, with average SDs of $0.07 \mathrm{~mm}$ (preoperatively), and $0.08 \mathrm{~mm}, 0.09 \mathrm{~mm}$, and 0.08 $\mathrm{mm}$ (at 7, 30, and 90 days postoperatively, respectively). We found significant overall flattening $(P<0.05)$ of the anterior cornea 1 week following surgery in 8 out of 10 patients (all patients except for $S \# 2$ and $S \# 3$ ), with an average radius increase by $2.25 \%$. In six of these patients there was further corneal flattening during the tested period, with an average radius increase by $5.5 \%$ at 90 days. $S \# 2$ and $S \# 3$ experienced an initial corneal steepening, followed by a corneal flattening, but the radius of curvature at 90 days was not statistically significantly different from preoperative values. Figure $4 \mathrm{~b}$ shows $\mathrm{Rx}$ to Ry ratio pre- and postoperatively $(7,30$, and 90 days). In 7 out of 10 patients (all except for $\mathrm{S} \# 2, \mathrm{~S} \# 7$, and

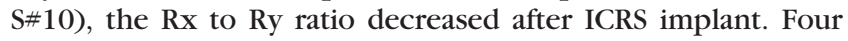




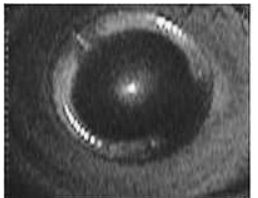

S\#1 (OS)

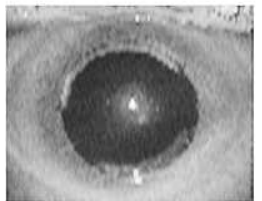

S\#6 (OD)

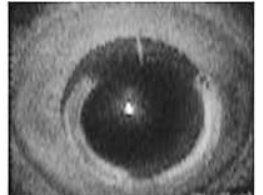

S\#2 (OS)

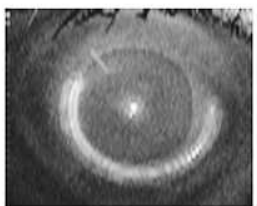

S\#7 (OS)

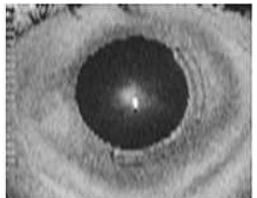

$\mathrm{S \# 3}$ (OD)

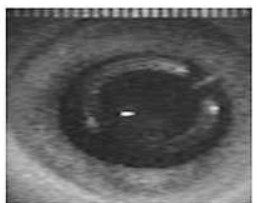

S\#8 (OS)

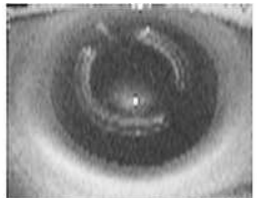

$\mathrm{S \# 4}$ (OS)

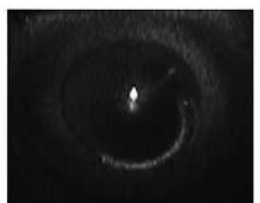

S\#9 (OD)

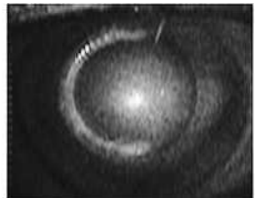

S\#5 (OS)

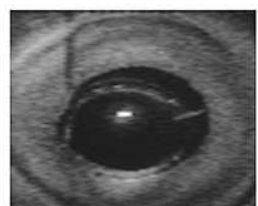

S\#10 (OS)

Figure 1. Enface OCT images in all patients, showing the lateral location of the ICRS

patients ( $\mathrm{S} \# 3, \mathrm{~S} \# 5, \mathrm{~S} \# 6$, and $\mathrm{S} \# 9)$ showed $\mathrm{Rx}$ to Ry ratio close to 1. On average, the difference in anterior curvature in the horizontal and vertical meridians decreased significantly from a mean preoperative value of $4.52 \pm 2.62 \mathrm{D}$ to a mean 90-day postoperative value of $2.81 \pm 2.39 \mathrm{D}(P<0.05)$.

The overall anterior corneal flattening ranged from $8.9 \%$ to $2.2 \%$ across patients. We found a tendency for $\mathrm{Rx}$ to flatten more than Ry. Rx flattening ranged from $18.6 \%$ to $1.5 \%$ across patients, and Ry from $0.8 \%$ to $9.1 \%$. Flattening in $\mathrm{Rx}$ was statistically significant at 7,30, and 90 days, whereas changes in Ry were not statistically overall.

Figure 5 shows the longitudinal variation of asphericity in the horizontal (Qx, Fig. 5a) and in the vertical (Qy, Fig. 5b). Preoperatively, patients showed typically a highly prolate horizontal meridian $(\mathrm{Qx}=-1.64 \pm 0.91)$ and much lower vertical negative aphericity (or even positive asphericity values) in the vertical meridian ( $\mathrm{Qy}=-0.11 \pm 0.72)$. In general, keratoconic patients showed higher magnitudes of asphericity ( $Q$ ranging from -3.65 to 0.72 ) in comparison with a normal population. ${ }^{28}$ The ICRS implant produced significant changes in Qx or Qy. On average, Qx shifted toward more negative values 7 days after the procedure (from -1.64 to 2.25 ), but typically decreased to values not significantly different from preoperative values $(\mathrm{Qx}=-1.49 \pm 1.02) 90$ days after the procedure. Qy did not follow a systematic pattern immediately after surgery or longitudinally.

Figure 6 shows anterior corneal elevation maps from two patients ( $\mathrm{A} \# 5$ and $\mathrm{S} \# 8$ ), for 4-mm diameters, centered at the pupil center. The radii of curvature of the best fitting spheres and the corneal elevation RMS for asymmetric terms (excluding astigmatism) are also shown. These patients show corneal flattening after surgery and during the follow up, as well as a reduction of the asymmetry of the corneal elevation map, with a decrease in the corneal elevation asymmetric RMS of $19.7 \%$ (S\#5) and 14.6\% (S\#8), respectively.

Figure 7 shows the anterior cornea RMS_asym, including astigmatism (Fig. 7a) and excluding astigmatism (Fig. 7b). On average RMS_asym did not show significant differences between pre- and postoperative measurements, primarily due to the high intersubject variability of the corneal elevation
(1) 3-D Raw data

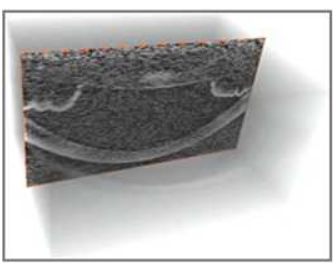

(6) Corneal topographies (S\#2)

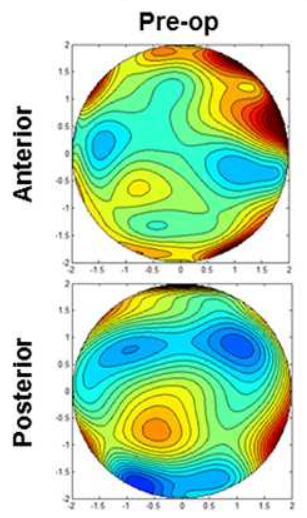

(2) Clustering
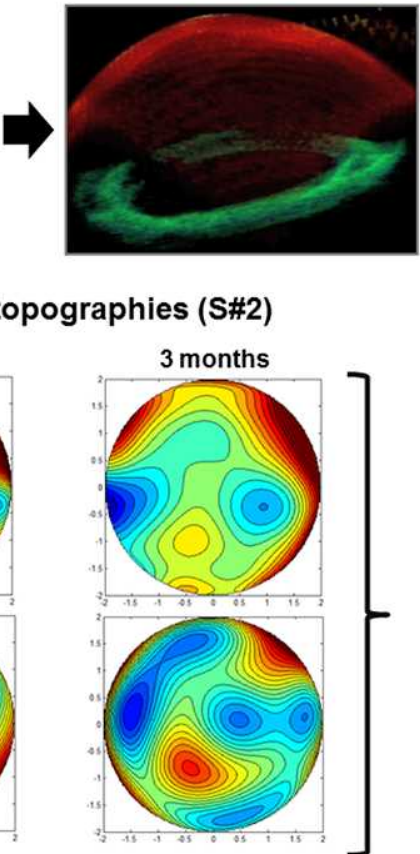

\section{(3) Multilayer segmentation}

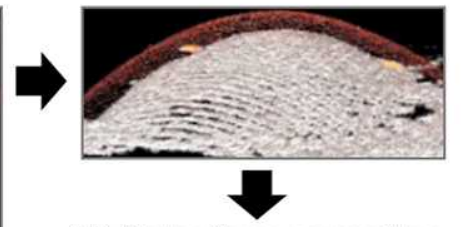

(4) Distortions correction

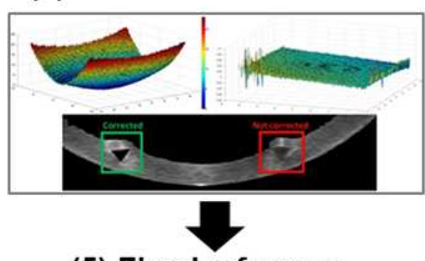

(5) Fixed reference

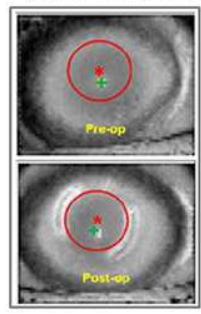

Figure 2. Illustration of the OCT image analysis and quantification in patient $\mathrm{S} \# 2$. 


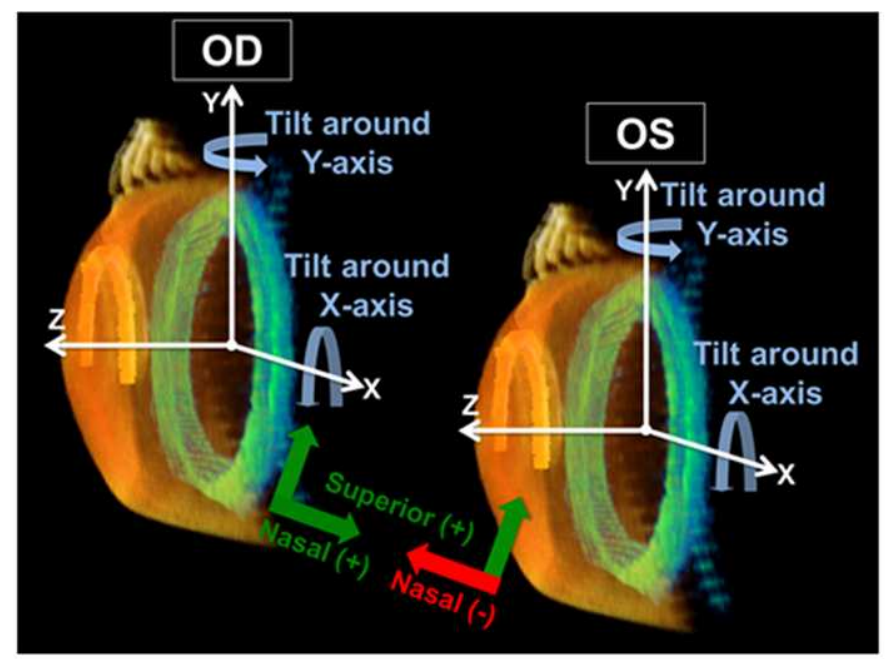

(a)

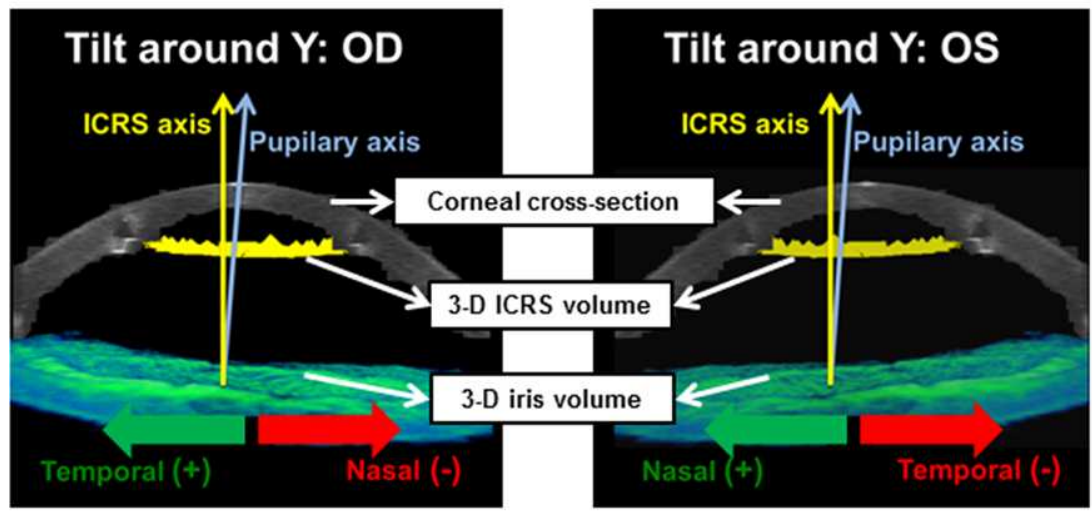

(b)

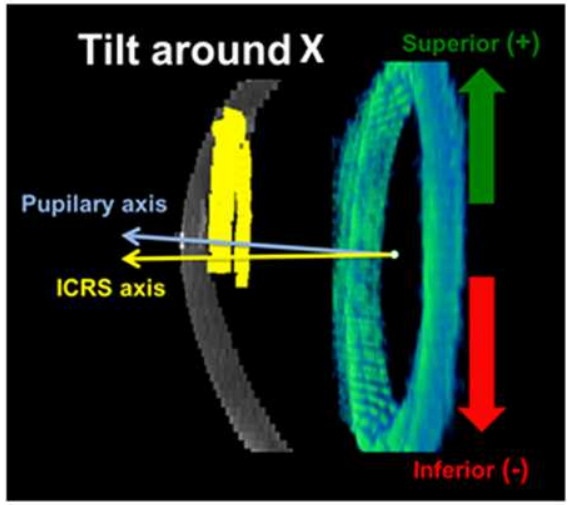

(c)

Figure 3. (a) Definition of pupillary plane (and axis), ICRS plane (and axis), and ICRS tilt. (b) Sign convention ICRS tilt around the $y$-axis, for OD and OS. (c) Sign convention of ICRS tilt around the $x$-axis.

asymmetry preoperatively. $\mathrm{S} \# 5, \mathrm{~S} \# 6$, and $\mathrm{S} \# 8$ experienced a decrease in asymmetry (with and without astigmatism) from preoperatively to 90 days postoperatively $(P<0.05)$. S\#3 and $\mathrm{S} \# 4$ increased asymmetry significantly $(P=0.05)$. Interestingly,

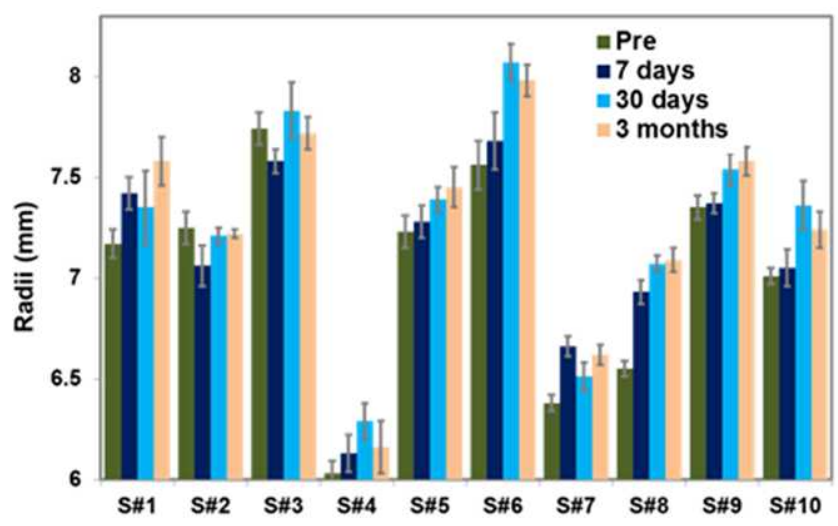

(a) the subject with highest amount of preoperative RMS_asym (S\#8) improved symmetry significantly (and progressively) both with and without astigmatism, whereas the subject with lowest preoperative RMS_asym (S\#3) increased asymmetry

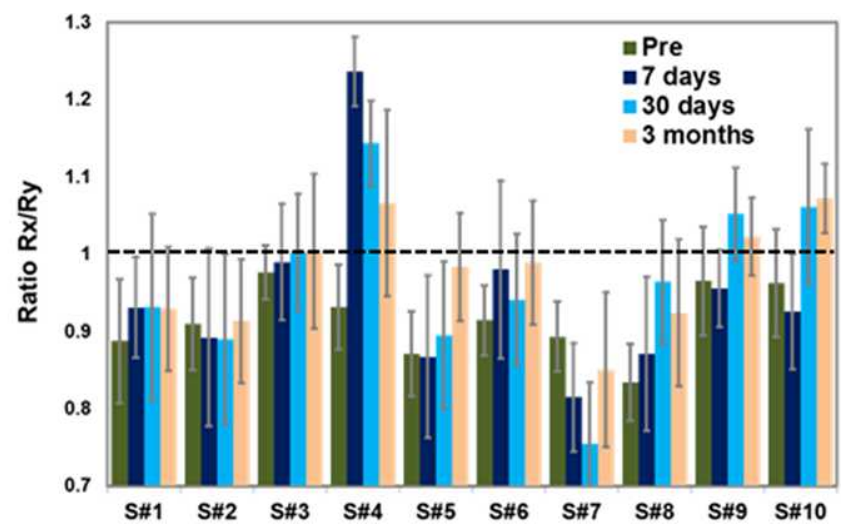

(b)

Figure 4. (a) Anterior cornea mean radius of curvature and (b) Rx to Ry ratio at various time points (pre- and 7, 30, and 90 days postoperatively). Data are for all patients. 


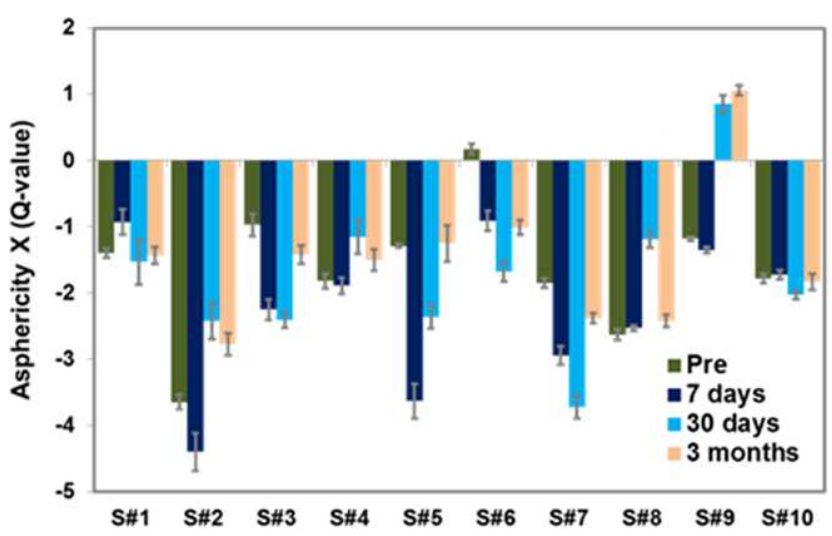

(a)

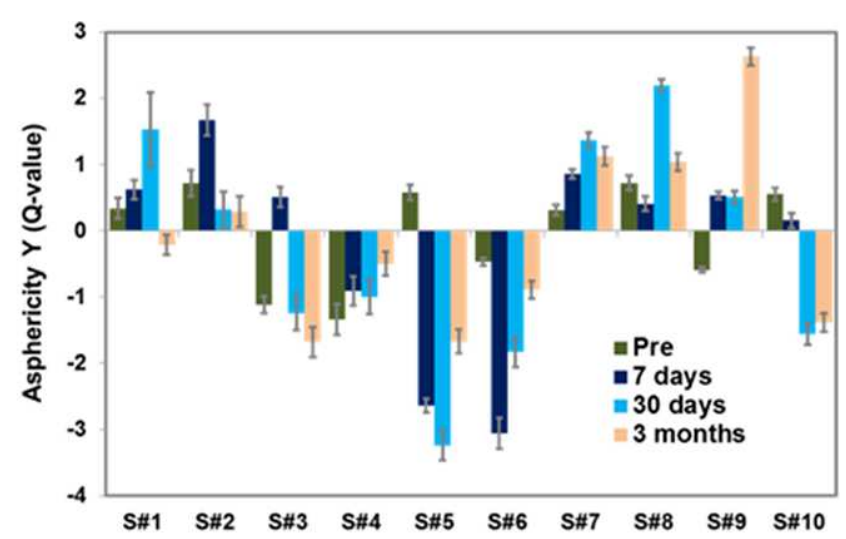

(b)

Figure 5. (a) Asphericity in the horizontal meridian (Qx) and (b) asphericity in the vertical meridian (Qy), pre- and 7, 30 , and 90 days postoperatively.

significantly. As expected, we found in the RMS_asym excluding astigmatism lower values $(P<0.001)$ in comparison with RMS_asym with astigmatism; however, the RMS (with and without astigmatism) show similar trends during the follow up.

\section{Longitudinal Changes of Posterior Corneal Surface Geometry and Topography}

The radius of curvature of the posterior corneal surface was obtained from sphere fitting, in a 4-mm optical zone. Intrasubject repeatability in the estimated anterior radius of curvature across repeated measurements is high, with average SDs of $0.08 \mathrm{~mm}$ (preoperatively) and $0.09 \mathrm{~mm}(7,30$, and 90 days postoperatively). Figure 8 shows the longitudinal varia- tions in radius of curvature of the posterior corneal surface with surgery. Unlike for the anterior surface, where most patients showed flattening, there was not a clear trend for the posterior surface. Only 3 patients ( $\mathrm{S} \# 1, \mathrm{~S} \# 4$, and $\mathrm{S} \# 9$ ) showed flattening of the posterior cornea (average: $3.7 \%$ at 7 days, $6.4 \%$ at 30 days, and $13.9 \%$ at 90 days), and $\mathrm{S} \# 2, \mathrm{~S} \# \mathbf{5}, \mathrm{S} \# 6, \mathrm{~S} \# \mathbf{8}$, and S\#10 showed steepening (average: $8.8 \%$ at 7 days, $6.7 \%$ at 30 days, and $6.9 \%$ at 90 days).

Figure 9 shows posterior corneal elevation maps from two patients ( $\$ \# 1$ and $\mathrm{S} \# 10$ ), for 4-mm diameters, centered at the pupil center. Maps are clearly dominated by astigmatism. Although the presence of ICRS induces marked changes in the topographic pattern with time, the posterior surface did not
Pre-op $\mathrm{R}=7.19 \mathrm{~mm} ; \mathrm{RMS}=6.84 \mu \mathrm{m}$

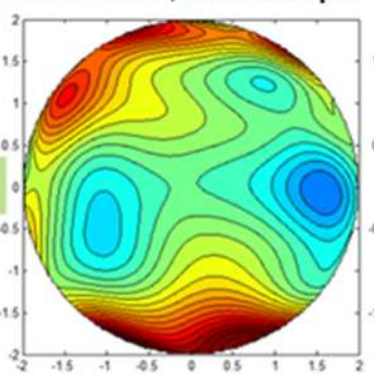

S\#5

S\#8

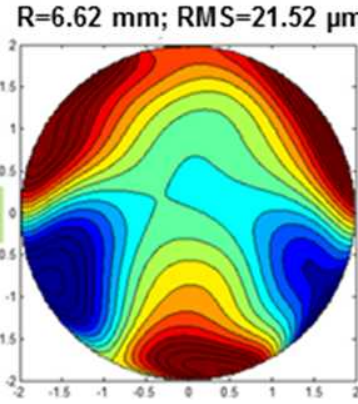

7 days

$\mathrm{R}=7.31 \mathrm{~mm} ; \mathrm{RMS}=8.53 \mu \mathrm{m}$
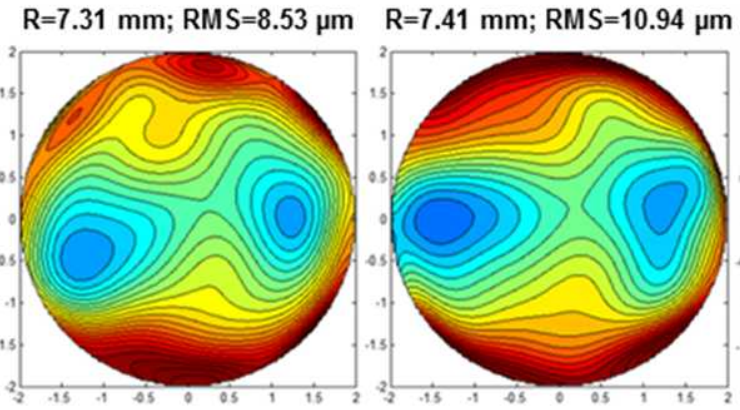

30 days

3 months

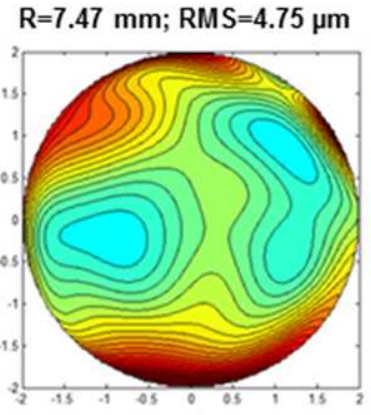

$(\mathrm{mm})$
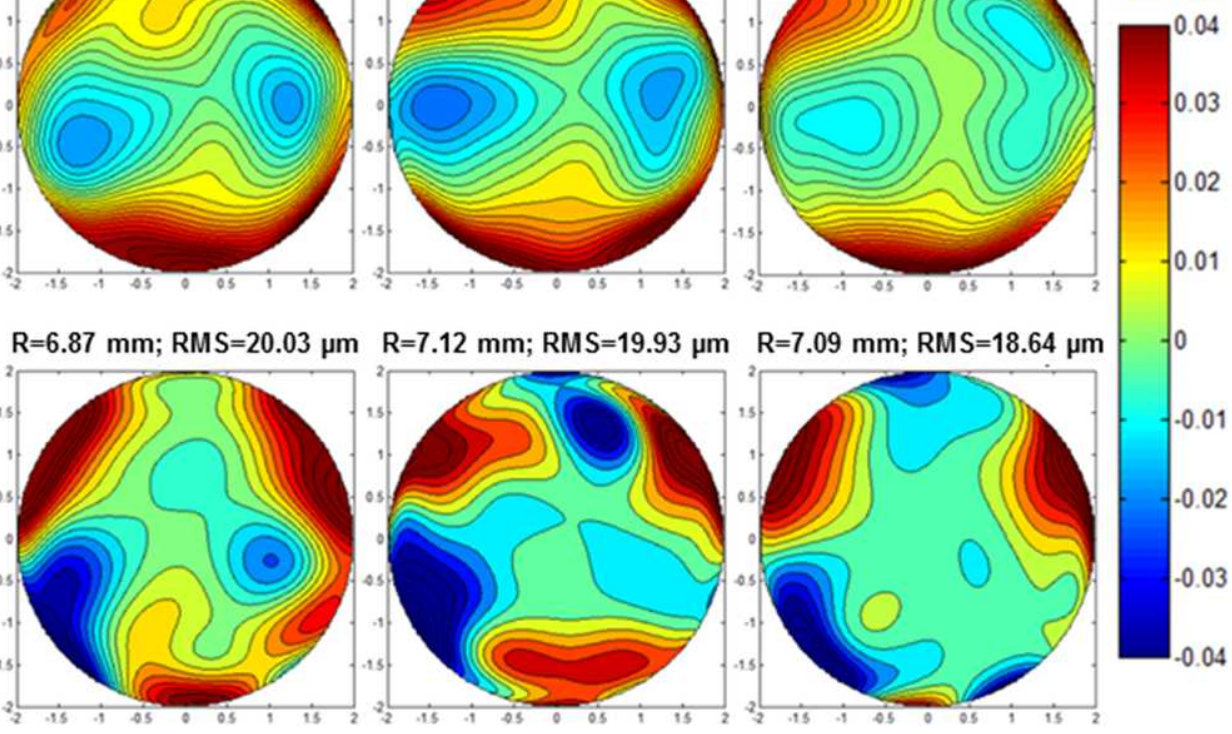

Figure 6. Anterior corneal elevation maps pre- and postoperatively in two patients of the study ( $\mathrm{S} \# 5$ and $\mathrm{S} \# 8$ ). $R$ stands for radius of curvature and RMS for RMS_asym. 


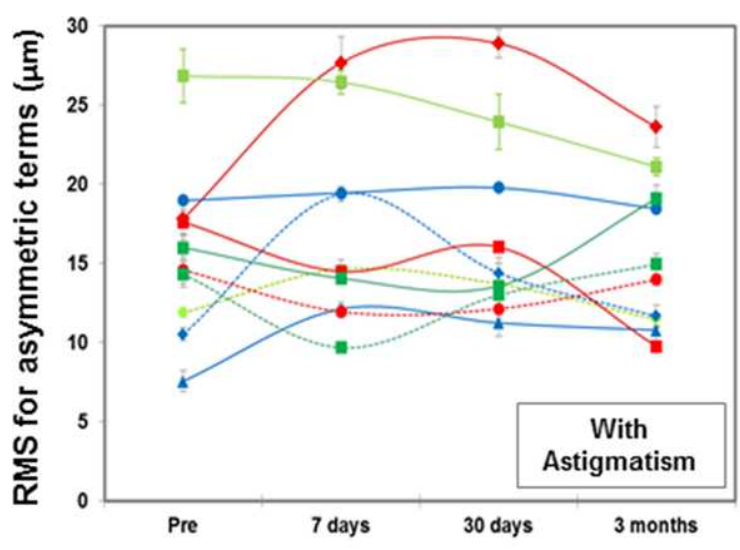

(a)

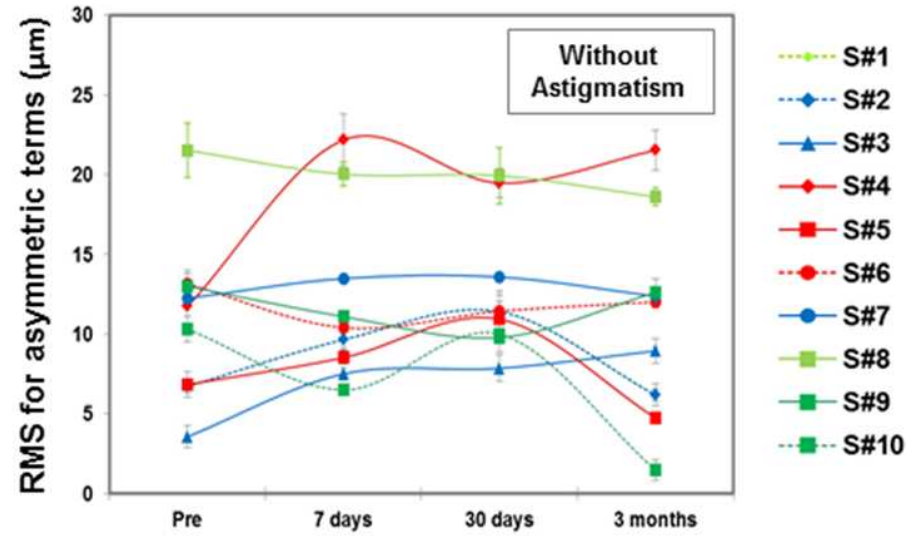

(b)

FiguRE 7. Anterior corneal elevation RMS for asymmetric Zernike coefficients (RMS_asym), (a) with astigmatism, and (b) without astigmatism, for all patients.

show significant decrease in astigmatism and asymmetric terms with surgery.

Figure 10 shows the posterior cornea RMS_asym, including astigmatism (Fig. 10a) and excluding astigmatism (Fig. 10b). ICRS produced significant changes although the longitudinal trends show high intersubject variability. On average RMS_asym including astigmatism showed a slight but not significant $(P=0.4)$ decrease (from $58.5 \pm 8.8$ to $54.1 \pm 6.8 \mu \mathrm{m}$, preoperatively to 90 days postoperatively). $\$ \# 4, S \# 7$, and $S \# 8$ experienced a decrease in RMS_asym (with and without astigmatism) from preoperatively to 90 days postoperatively $(P<0.05)$. S\#1, S\#5, and $\$ \# 9$ increased asymmetry significantly $(P<0.05)$. Interestingly, the patients with highest amount of preoperative asymmetric RMS ( $\mathrm{S} \# 7$ and $\mathrm{S} \# 8$ ) improved symmetry significantly (and progressively).

\section{Longitudinal Variations of Corneal Power}

Refractive changes are associated with changes in curvature of both the anterior and posterior corneal surfaces. Figure 11 shows the corneal power change during the follow-up (Fig. 11a), and the preoperatively versus postoperatively ( 90 days) corneal power (Fig. 11b). Corneal refractive power changed significantly from a mean preoperative value of $46.2 \pm 3.2 \mathrm{D}$ to a mean 90-day postoperative value of $44.4 \pm 3.5 \mathrm{D}(P<0.05)$. On average, corneal power decreased $1.71 \mathrm{D}$ (between $-5.1 \mathrm{D}$

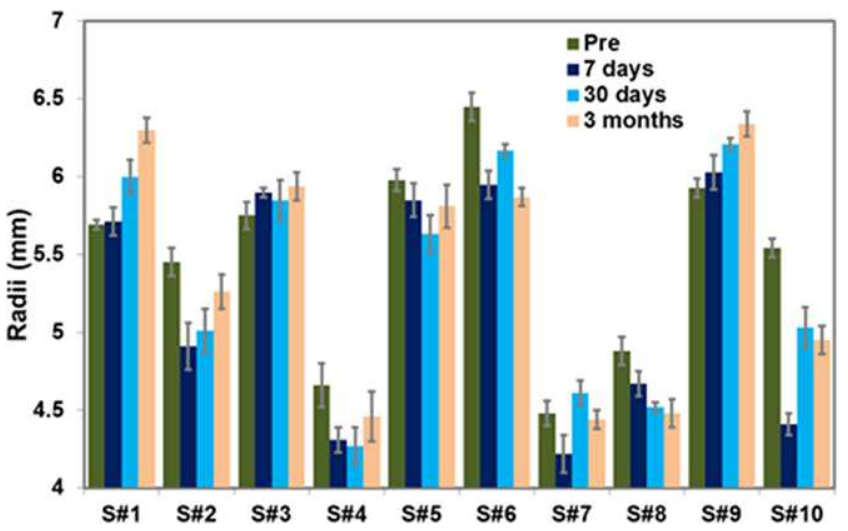

FIGURE 8. Posterior corneal mean radius of curvature (pre- and postoperatively 7-90 days), in all patients of the study. for $\mathrm{S} \# \mathbf{8}$ to $+1.1 \mathrm{D}$ for $\mathrm{S} \# 4$ ). Preoperative corneal power is highly correlated with postoperative power $(P<0.05$; Fig. 11b).

\section{Corneal Thickness: Pre- and Post-ICRS Implantation}

Figure 12a shows an example ( $\mathrm{S} \# 2)$ of the corneal thickness map pre- and postoperatively. Minimum corneal thickness did not change significantly with surgery $(384 \pm 60 \mu \mathrm{m}$ preoperatively to $396 \pm 46 \mu \mathrm{m}$ postoperatively). However, both the distribution of corneal thickness and changes in the minimum thickness location did occur. Figure $12 \mathrm{~b}$ shows the longitudinal variation of the RMS of the corneal thickness map (RMS_thicknessmap) for all patients of the study as an estimation of the thickness distribution in the 4-mm optical zone. Corneal thickness redistribution occurred in most patients with time. RMS_thicknessmap decreased significantly (from preoperatively to 90 days postoperatively, $P<0.05$ ) in three subjects ( $\$ \# 4, S \# 7$, and $S \# 9$ ), and increased significantly $(P<0.05)$ in three patients $(\mathrm{S} \# 2, \mathrm{~S} \# 8$, and $\mathrm{S} \# 10)$. Figure $12 \mathrm{c}$ shows the displacement of the minimum corneal thickness location across the optical zone, for all patients. Overall, there is an average displacement of the location of minimum thickness from inferior preoperatively (centroid coordinates: $-0.01[x$-axis $]$ and $-0.63[y$-axis $])$ toward more central postoperatively (centroid coordinates: $-0.06[x$-axis $]$ and -0.14 [ $y$-axis] at 90 days). The largest shift occurred between preoperatively and 7-days postoperatively, with little changes during the follow-up.

\section{D ICRS Location}

The location of the ICRS was analyzed in $3 \mathrm{D}$, both in terms of the implantation depth and tilt. Figure 13a shows the ICRS depth (computed from the average distance from anterior corneal surface to the center of mass of the ICRS at every location along the ICRS) in all patients, at different times postoperatively. On average, there was a slight, but progressive, decrease of ICRS depth (by $10 \mu \mathrm{m}$, from 7-90 days postoperatively). Most patients showed longitudinal changes in ICRS depth. Patients S\#2 (Fig. 13), S\#6 (Fig. 13), and $S \# 9$ and $S \# 10$ (Fig. 13) showed a mean forward shift of the ICRS of $18 \pm 6 \mu \mathrm{m}$; and $\mathrm{S} \# 1$ (Figs. 13), S\#6 (Fig. 13), S\#7, S\#8 (Fig. 13) showed a backward shift of the ICRS (41 $\pm 17 \mu \mathrm{m})$ at 90 days. Figure $13 \mathrm{~b}$ shows the correlation between the ICRS planned depth and the measured ICRS 


\section{Pre-op}

7 days

30 days

\section{3 months}
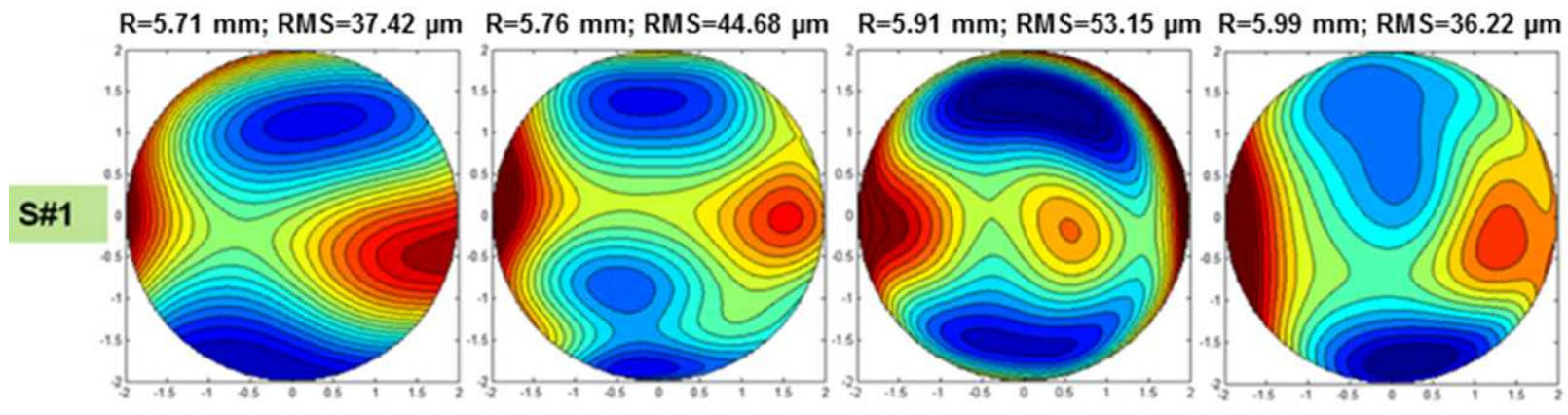

(mm)
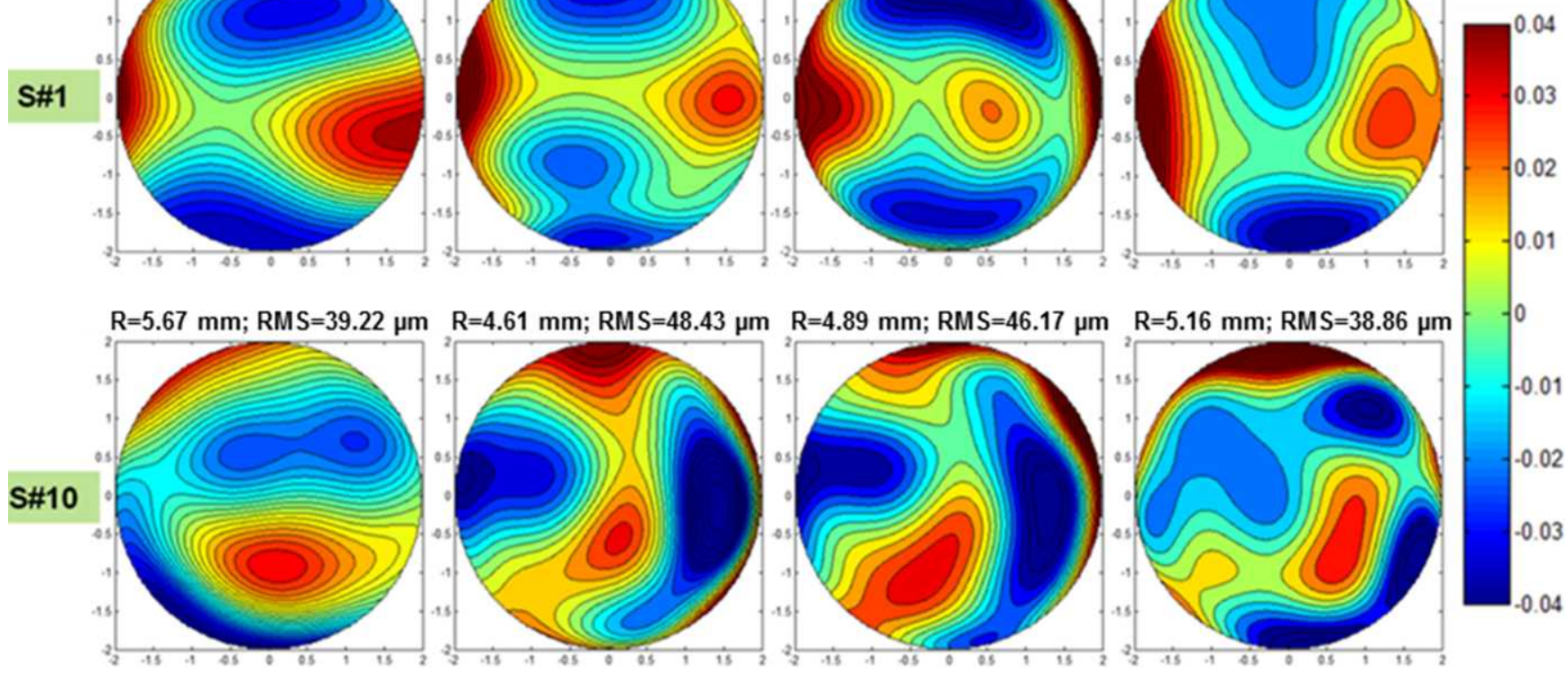

Figure 9. Posterior corneal elevation maps pre- and postoperatively in two patients of the study (S\#1 and S\#10). $R$ stands for radius of curvature and RMS for RMS_asym.

depth at 7 days. The correspondence between the planned and the measured ICRS depth is higher for the femtosecond technique $(15 \pm 20 \mu \mathrm{m}$ between the planned and achieved depth) than for the manual technique $(40 \pm 22 \mu \mathrm{m}$ difference).

Figure 14 shows the ICRS tilt angles around $\mathrm{x}$ and $\mathrm{y}$-axes in all patients and postoperative time points. The tilt angles of the left and right ICRS segments have been changed in sign for the nasal/temporal coordinates, to allow appropriate averaging. On average, there is a forward tilt of the temporal and superior part of the ring, with an overall tilt of $-6.8 \pm 2.6^{\circ}$ (temporal) and $-2.1 \pm 0.8^{\circ}$ (superior) at 7 days. Although there is intersubject variability, on average, there is small $\left(<1^{\circ}\right)$ but systematic tilt of the ICRS between 7 and 90 days postsurgery.

\section{Correlation Between Surgical Parameters and Corneal Geometrical Response}

The effect of ICRS implantation on corneal curvature was highly dependent on ICRS diameter. The ICRS with smaller diameters were more effective in flattening the anterior corneal surface than larger diameters: $0.33 \pm 0.18 \mathrm{~mm}(5$ $\mathrm{mm}$ optical zone) vs. $0.17 \pm 0.14 \mathrm{~mm}$ (6-mm optical zone), at 90-days postoperatively. As a result, the 5-mm ICRS produced the largest changes in corneal power. For example, $\mathrm{S} \# \mathbf{8}$, with a 5-mm optical zone, experienced a decrease in total corneal power by $5.1 \mathrm{D}$ (90-days postoperatively).

The optical zone diameter appeared also correlated, to a less extent, to the change of the posterior corneal surface radius of curvature. A 5-mm optical zone diameter produced an

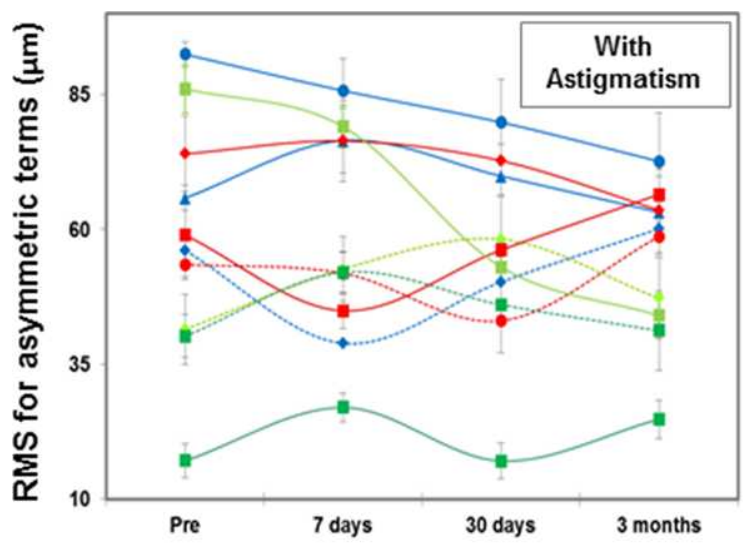

(a)

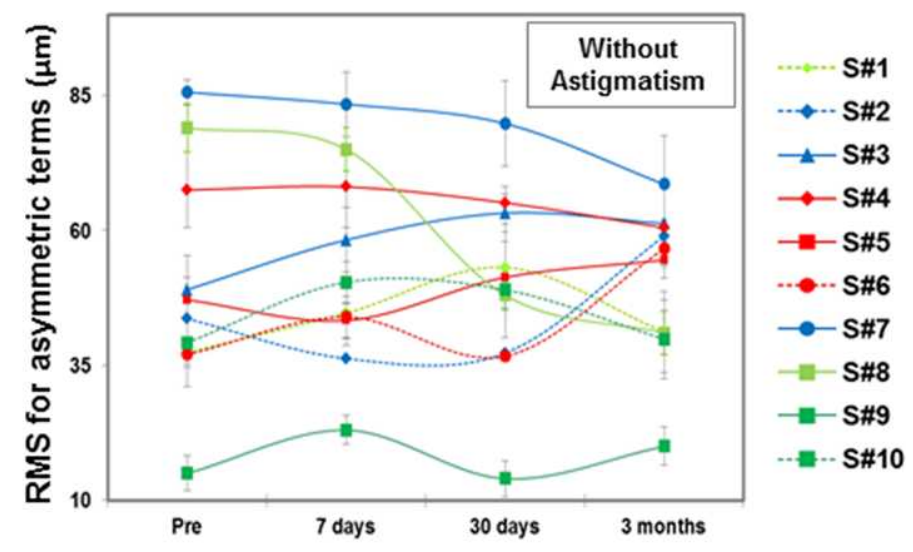

(b)

FIgURE 10. Posterior corneal elevation RMS for asymmetric Zernike coefficients (RMS_asym), (a) with astigmatism, and (b) without astigmatism, for all patients. 


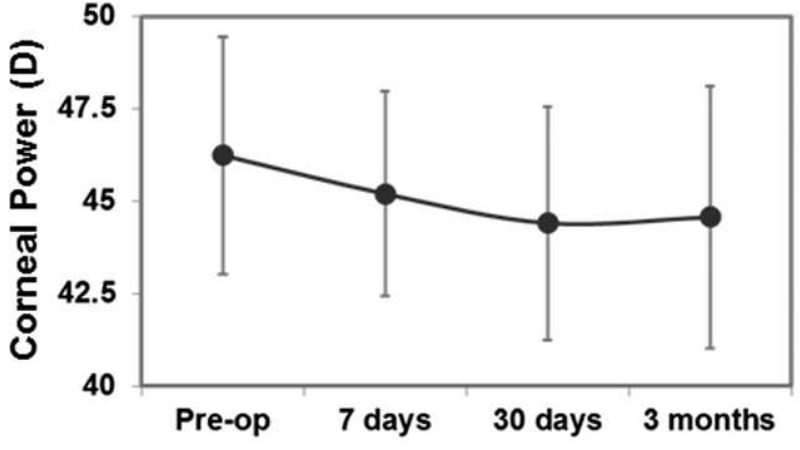

(a)

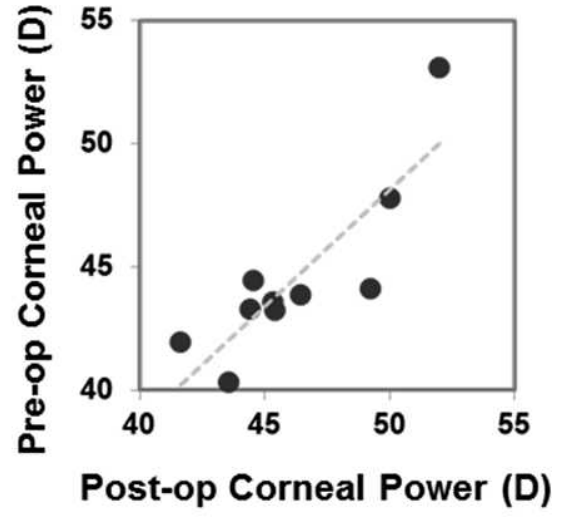

(b)

Figure 11. (a) Corneal power change during the follow-up; (b) preoperative versus postoperative (90 days) corneal power.

average posterior corneal flattening of $0.14 \pm 0.45$ whereas a 6-mm optical zone diameter produced an average steepening of $0.31 \pm 0.25 \mathrm{~mm}$ (90-days postoperatively). In addition, the effect of ICRS on the posterior surface could be possibly associated to the ICRS arc length. Previously, we described steepening on the posterior corneal surface with a $90^{\circ}$ arc length. ${ }^{29}$ A combination of $90^{\circ}$ arc length ICRS and $160^{\circ}$ arc length (as in patients $\mathrm{S} \# 4, \mathrm{~S} \# 6, \mathrm{~S} \# 8$, and $\mathrm{S} \# 10$ ) produced a significant steepening of the posterior corneal surface (by 0.41 $\pm 0.24 \mathrm{~mm}$ ). However, a combination of $160^{\circ}$ and $120^{\circ}$ arc length ICRS (as in patients $S \# 1, S \# 2, S \# 3$, and $S \# 9$ ) tended to flatten the posterior corneal surface (by $0.15 \pm 0.35 \mathrm{~mm}$ ). In addition, the ICRS arc length seems to be associated with the postoperative corneal symmetry. Patients implanted with $90^{\circ}$
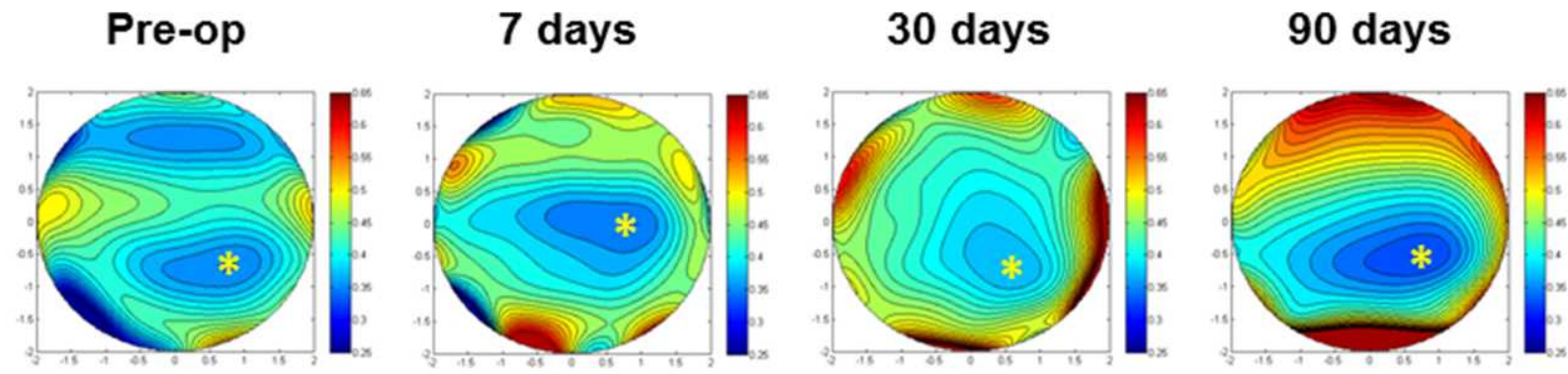

(a)

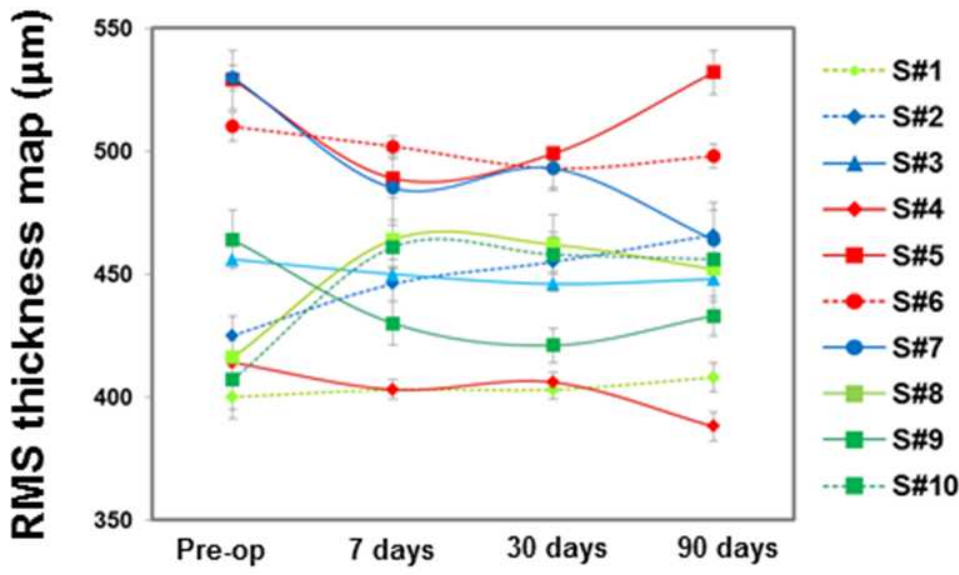

(b)

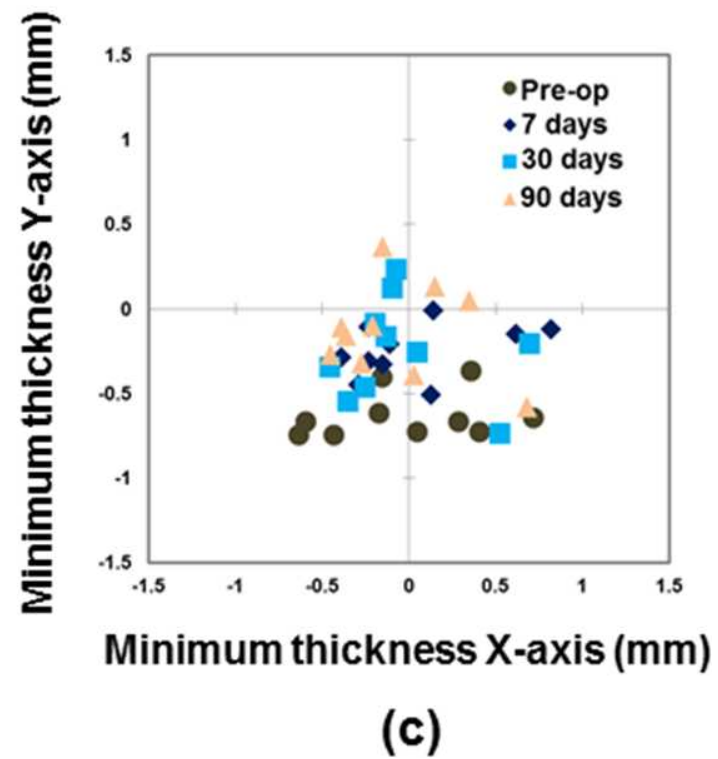

FiguRE 12. (a) Thickness map for $\mathrm{S} \# 2$ preoperatively, and 7, 30, and 90 days postoperatively. Asterisks denote the minimum corneal thickness location. (b) RMS of the corneal thickness map. (c) Coordinates of the minimum corneal thickness location, preoperatively, and 7, 30, and 90 days postoperatively for all patients. 


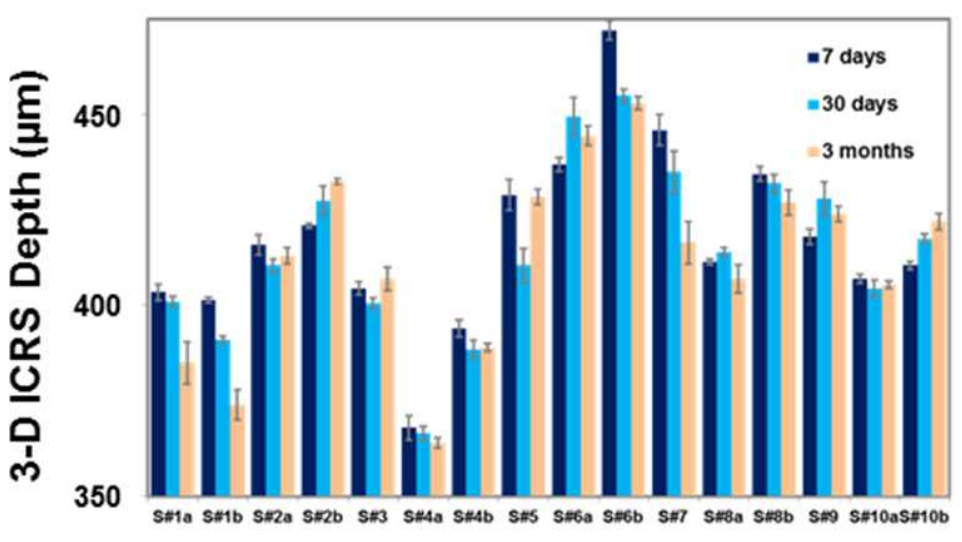

(a)

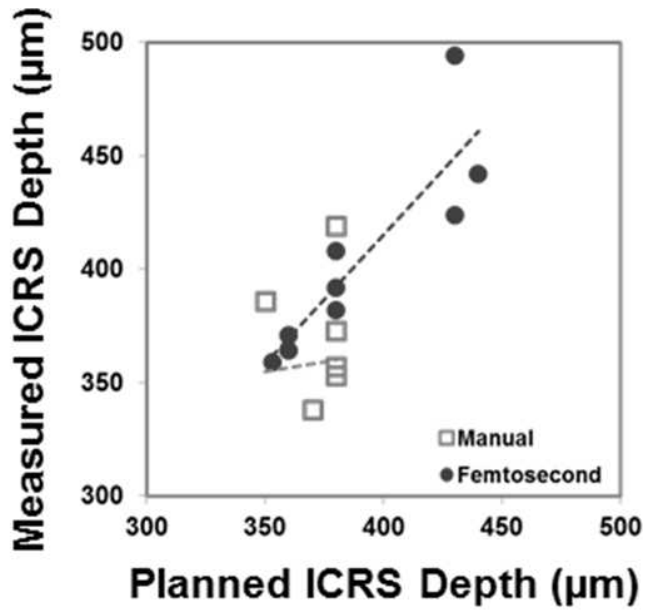

(b)

FIGURE 13. (a) Average ICRS depth for all patients (7, 30, and 90 days postoperatively). (b) Planned depth versus OCT measured depth.

arc length ICRS showed a significant decrease of the corneal surface RMS for asymmetric terms of the posterior surface (from $63.4 \pm 8.8 \mu \mathrm{m}$ preoperatively to $51.8 \pm 6.0 \mu \mathrm{m}$ 90-days postoperatively) and a slight increase in the RMS of the thickness map (from $436 \pm 8 \mu \mathrm{m}$ preoperatively to $448 \pm 9$ $\mu \mathrm{m} 90$ days postoperatively).

The position and rotation of the ICRS also seem to play a major role in the symmetry of the postoperative cornea. A strong direct correlation was found between the change in the ICRS tilt around $x$ and change in anterior corneal RMS_assym, between 7 and 90 days ( $r=+0.83, P<0.05$ with astigmatism; $r$ $=+0.76, P<0.05$ without astigmatism). No significant correlations were noted among other parameters of the ICRS position and the corneal surface.

\section{Discussion}

We have presented a longitudinal study for the 3D geometrical characterization of both anterior and posterior corneal surfaces

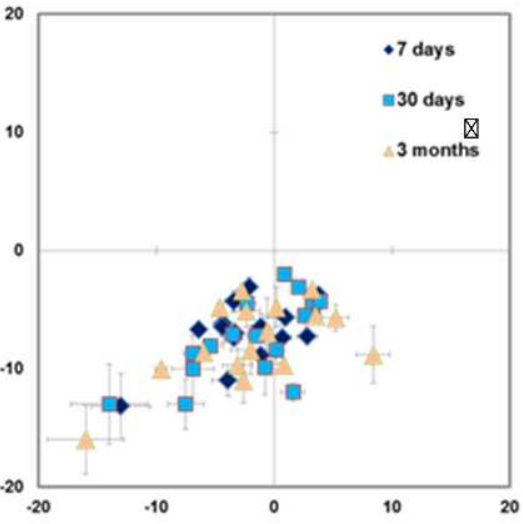

Rotational Angle aroundX (deg)

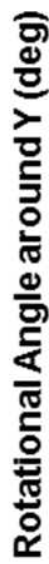

엉 (a) superior segment and [b] right/inferior segment). in keratoconus following ICRS implantation (in three time points from 7-90 days postoperatively). Besides geometrical changes, we have also quantified three-dimensionally ICRS positioning with time. This study is, to our knowledge, the first attempt for full quantification of geometric, topographic, and pachymetric corneal changes following ICRS surgery, and 3D ICRS accurate location based on distortion corrected OCT.

Accurate measurements of anterior and posterior corneal topographies are essential to understand the corneal response to ICRS implants. Several studies have used Scheimpflug and ultrasound biomicroscopy in keratoconus eyes post ICRS surgery, but these techniques are challenged by resolution, distortions and a sparse sampling of the cornea. ${ }^{35-39}$ To our knowledge, the effect of ICRS on the posterior corneal surface has only been investigated recently using Scheimpflug imaging. ${ }^{40}$ The higher speed and resolution, axial and lateral, of sOCT makes of this an ideal tool to evaluate corneal geometry in keratoconus and its ICRS treatment. Most studies of keratoconus address only the measurement of corneal thickness and corneal radii, ${ }^{16-19}$ while few attempt quantifica-

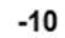

$-20-10$

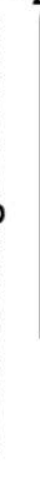

(b)

FiguRE 14. (a) Average ICRS tilt for all patients at 7, 30, and 90 days. (b) ICRS tilt for patients with two segments at 7, 30, and 90 days ([a] left/ 
tion of corneal topography. ${ }^{20,21}$ In fact, reports comparing corneal shape (radii and pachymetry) measured with OCT and other techniques are conflicting, with some of the studies showing significant differences found across instruments, ${ }^{21}$ and others ${ }^{20}$ reporting a good agreement between OCT and Scheimpflug.

Due to the scanning configuration and the refraction effects (particularly relevant in this case due to the ICRS inside the cornea with a higher index of refraction), fan and optical distortion affect significantly the acquisition of accurate quantitative 3D data from OCT corneal surfaces. ${ }^{25-28}$ In previous studies, Ortiz et al. ${ }^{29,33,34}$ described the application of custom algorithms for reconstructing accurately 3D corneal elevation maps after fan and optical distortion correction. The accuracy of these algorithms and the automatic image processing tools have been demonstrated previously, ${ }^{25-28}$ and applied to the quantification of corneal geometry in keratoconus before and after ICRS surgery and ICRS locatization. ${ }^{29}$

ICRS act as spacer elements between the bundles of corneal lamellae, inducing shortening of the central corneal arc length and, as consequence, producing a flattening of the anterior cornea. ${ }^{3,4}$ Most studies analyzed changes in the anterior cornea only, with very few studies addressing the posterior cornea. ${ }^{3-6,39,41}$ Previous studies reported a mean flattening of the anterior corneal surface by 2.5 D. ${ }^{5,6}$ Ortiz et al. ${ }^{29}$ reported a decrease of corneal power (considering the anterior and posterior corneal surfaces) of $2.62 \mathrm{D}$ in a single subject, using OCT. Also, in this study we found a mean decrease of corneal power (anterior + posterior corneal surfaces) of $1.71 \pm 1.83 \mathrm{D}$ 90 days postoperatively. Despite the overall decrease of corneal power, the response varied across individuals (ranging from an increase of $1.07 \mathrm{D}$ to a decrease of $5.10 \mathrm{D}$ at 90 days). This intersubject variability is consistent with a previous study using videokeratography, who found an increase of $2.5 \%$ to a decrease of $18 \%$ in the topographic K-values, in 21 eyes 90 days post implantation of ICRS. ${ }^{5}$ As predicted by a recently published Finite Element Model analysis of the corneal response to ICRS implants, we found that the change in anterior corneal radius was highly dependent on the optical zone diameter. ${ }^{32}$

Regarding the effect of ICRS on the posterior corneal surface, a previous study based on Scheimpflug imaging reported a significant flattening of approximately $0.25 \mathrm{~mm}$ after ICRS implantation. ${ }^{40}$ In the current study, we found that the posterior corneal radii flattened in four eyes (by $0.59 \pm$ $0.41 \mathrm{~mm}$ ), but steepened in six eyes (by $0.32 \pm 0.22 \mathrm{~mm}$ ) 90 days postoperatively. This variable response appears to be associated with the arc length of the ICRS, with the combination of $90^{\circ}$ and $160^{\circ}$ arc lengths ICRS producing a significant steepening on the posterior corneal surface. The optical zone diameter appeared to play also some role in the posterior corneal changes.

The regularity of both anterior and posterior corneal surfaces was analyzed by means of RMS of the corneal elevation maps asymmetric terms. In agreement with Chen and Yoon, ${ }^{42}$ who had reported that the posterior corneal surface profile is more irregular than that of the anterior corneal surface in keratoconus, we found much higher preoperative RMS_assym in the posterior corneal surface than in the posterior corneal surface $(50.0 \pm 21.4 \mu \mathrm{m}$ vs. $10.6 \pm 5.3$ $\mu \mathrm{m})$. We found that the ICRS did not systematically reduced RMS_assym in either surface. The thickness redistribution after ICRS implantation has been suggested as a delay factor in keratoconus progression of the disease, since as cornea thickens in the weakest areas, the stress may be redistributed and the decompensatory biomechanical cycle might be delayed. ${ }^{7}$ We did not find a systematic increase in the minimum thickness or in the corneal thickness regularity, although the location of minimum thickness tended to move more centrally postsurgery.

Finally, the automatic ICRS volume segmentation allowed a comprehensive characterization of the implanted ICRS. Migration, rotation, or extrusion of the ICRS has been related to surgical complications. ${ }^{30,31}$ During the first month following ICRS, the wound healing response remains active, and the increase of myofibroblasts in the ICRS edges may result in slight variations in ICRS position with time. Previous works have used OCT to characterized ICRS depth, but did not corrected for optical distortion, and the depth quantification was typically done by analyzing only a few cross-sectional OCT images. ${ }^{22-24}$ Naftali and Jabaly-Habib ${ }^{24}$ reported significant differences between the planned and measured ICRS depth $(\sim 120 \mu \mathrm{m})$. In this study, we showed for the first time systematic measurements of the position of the ICRS in 3D. Our measurements showed a very good agreement between the planned depth and the 3D expected depth $(\sim 24 \mu \mathrm{m}$, on average across all patients of the study, 7-days postoperatively), with a higher difference for the manual than for the femtosecond tunnel technique. The quantification of the ICRS in $3 \mathrm{D}$ also allowed a longitudinal analysis of the ICRS rotational angles. ICRS rotation appears to have a major impact on anterior corneal symmetry, given the correlation between the ICRS tilt around $x$ and change in RMS_assym for anterior cornea.

To sum up, this study presents the first report of comprehensive longitudinal quantification of ICRS surgery based on OCT, including anterior and posterior corneal geometrical, topographic, and pachymetric analysis, and 3D location (depth and rotation) of the ICRS. We have shown that ICRS produced a significant flattening of the anterior corneal surface, particularly with a $5-\mathrm{mm}$ optical zone diameter. There was a not a systematic improvement in corneal symmetry, which was influenced by the arc length of the ICRS, and by the ICRS rotation inside the cornea. Although the results are promising, further studies on a larger keratoconus population treated using similar surgical parameters may give further insights on the mechanism of action and help in improving surgical nomograms. The combination of accurate quantitative OCT data and mechanical modeling may provide a better approach for the improvement of ICRS surgery.

\section{Acknowledgments}

Supported by funding from the European Research Council under the European Union's Seventh Framework Programme (FP7/20072013)/European Research Council Grant Agreement Number 294099. This study has been also supported by Ministerio de Educación y Ciencia, Spain (Grants FIS2011-25637, CEN-2091021), and European Union Grant EURHORCs-ESF (SM). Authors also acknowledge Unidad Asociada IO-CSIC/FJD. Conflict of Interest: Spanish patent: Procedure to calibrate and correct the scan distortion of an Optical Coherence Tomography system, P201130685 (Sergio Ortiz, Susana Marcos, Damian Siedlecki and Carlos Dorronosoro).

Disclosure: P. Pérez-Merino, None; S. Ortiz, P; N. Alejandre, None; I. Jiménez-Alfaro, None; S. Marcos, P

\section{References}

1. Rabinowitz YS. Keratoconus. Surv Opbthalmol. 1998;42:297319.

2. Nordan LT. Keratoconus: diagnosis and treatment. Int $O p b$ thalmol Clin. 1997;37:51-63.

3. Colin J, Cochener B, Savary G, Malet F. Correcting keratoconus with intracorneal rings. J Cataract Refract Surg. 2000;26: 1117-1122. 
4. Siganos D, Ferrara P, Chatznikolas K, Bessis N, Papastergiou G. Ferrara intrastromal corneal rings for the correction of keratoconus. J Cataract Refract Surg. 2002;28:1947-1951.

5. Shabayek MH, Alió JL. Intrastromal corneal ring segment implantation by femtosecond laser for keratoconus. Ophthalmology. 2007;114:1643-1652.

6. Piñero DP, Alio JL, Teus MA, Barraquer RI, Uceda-Montañés A. Modelling the intracorneal ring segement effect in keratoconus using refractive, keratometric and aberrometric data. Invest Ophthalmol Vis Sci. 2010;51:5583-5591.

7. Dauwe C, Touboul D, Roberts CJ, et al. Biomechanical and morphological corneal response to placement of intrastromal corneal ring segments for keratoconus. J Cataract Refract Surg. 2009;35:1761-1767.

8. Torquetti L, Ferrara P. Intrastromal corneal ring segment implantation for ectasia after refractive surgery. $J$ Cataract Refract Surg. 2010;36:986-990.

9. Reinstein DZ, Gobbe M, Archer TJ, Silverman RH, Coleman DJ. Epithelial, stromal, and total corneal thickness in keratoconus: three-dimensional display with Artemis very-high frequency digital ultrasound. J Refract Surg. 2010;26:259-271.

10. Reinstein DZ, Srivannaboon S, Holland SP. Epithelial and stromal changes induced by Intacs examined by threedimensional very high-frequency digital ultrasound. $J$ Refract Surg. 2001;17:310-318.

11. Shankar H, Taranath D, Santhirathelagan CT, Pesudovs K. Repeatability of corneal first-surface wavefront aberrations measured with Pentacam corneal topography. J Cataract Refract Surg. 2008;34:727-734.

12. Read SA, Collins MJ, Iskander DR, Davis BA. Corneal topography with Scheimpflug imaging and videokeratography: comparative study of normal eyes. J Cataract Refract Surg. 2009;35:1072-1081.

13. Dutta D, Rao HL, Addepalli UK, Vaddavalli PK. Corneal thickness in keratoconus: comparing optical, ultrasound, and optical coherence tomography pachymetry. Ophthalmology. 2012;120:457-463.

14. Huang D, Swanson EA, Lin CP, et al. Optical coherence tomography. Science. 1991;254:1178-1181.

15. Grulkowski I, Gora M, Szkulmowski M, et al. Anterior segment imaging with spectral OCT system using a high-speed CMOS camera. Opt Express. 2009;17:4842-4858.

16. Li Y, Shekhar R, Huang D. Corneal pachymetry mapping with high-speed optical coherence tomography. Ophthalmology. 2006;113:792-799.

17. Li Y, Meisler DM, Tang M, et al. Keratoconus diagnosis with optical coherence tomography pachymetry mapping. $O p h-$ thalmology. 2008;115:2159-2166.

18. Li Y, Tang M, Zhang X, Salaroli CH, Ramos JL, Huang D. Pachymetric mapping with Fourier-domain optical coherence tomography. J Cataract Refract Surg. 2010;36:826-831.

19. Tang M, Chen A, Li Y, Huang D. Corneal power measurement with Fourier-domain optical coherence tomography. J Cataract Refract Surg. 2010;36:2115-2122.

20. Karnowski K, Kaluzny BJ, Szkulmowski M, Gora M, Wojtkowski M. Corneal topography with high-speed swept source OCT in clinical examination. Biomed Opt Express. 2011;2:27092720.

21. Szalai E, Berta A, Hassan Z, Módis L Jr. Reliability and repeatability of swept-source Fourier-domain optical coherence tomography and Sceimpflug imaging in keratoconus. $J$ Cataract Refract Surg. 2012;38:485-494.

22. Lai MM, Tang M, Andrade EM, et al. Optical coherence tomography to assess intrastromal corneal ring segments depth in keratoconic eyes. $J$ Cataract Refract Surg. 2006;32: 1860-1865.
23. Gorgun E, Kucumen RB, Ynerel NM, Ciftci F. Assessment of intrastromal ring segment position with anterior segment optical coherence tomography. Ophthalmic Surg Lasers Imaging. 2012;43:214-221.

24. Naftali M, Jabaly-Habib H. Depth of intrastromal corneal ring segments by OCT. Eur J Ophthalmol. 2013;23:171-176.

25. Ortiz S, Siedlecki D, Grulkowski I, et al. Optical distortion correction in optical coherence tomography for quantitative ocular anterior segment by three-dimensional imaging. Opt Express. 2010;18:2782-2796.

26. Zhao M, Kuo AN, Izzat JA. 3D refraction correction and extraction of clinical parameters from spectral domain optical coherence tomography of the cornea. Opt Express. 2010;18: 8923-8936.

27. Ortiz S, Siedlecki D, Remon L, Marcos S. Three-dimensional ray tracing on Delaunay-based reconstructed surfaces. Appl Opt. 2009;48:3886-3893.

28. Ortiz S, Siedlecki D. Pérez-Merino P, et al. Corneal topography from spectral optical coherence tomography (sOCT). Biomed Opt Express. 2011;2:3232-3247.

29. Ortiz S, Pérez-Merino P, Alejandre N, Gambra E, Jimenez-Alfaro I, Marcos S. Quantitative OCT-based corneal topography in keratoconus with intracorneal ring segments. Biomed Opt Express. 2012;3:814-825.

30. Samini S, François-Leger F, Touboul D, Colin J. Histopathological findings after intracorneal ring segments (INTACS) implantation in keratoconic human corneas. J Cataract Refract Surg. 2007;33:247-253.

31. Pérez-Merino P, Parra F, Ibares-Frías L, et al. Clinical and pathological effects of different acrylic intracorneal ring segments in corneal additive surgery. Acta Biomater. 2010;6: 2572-2579.

32. Kling S, Marcos S. Finite element modeling of intra-stromal ring segment implantation into a hyperelastic cornea. Invest Ophthalmol Vis Sci. 2013;54:881-889.

33. Ortiz S, Pérez-Merino P, Gambra E, de Castro A, Marcos S. In vivo human crystalline lens topography. Biomed Opt Express. 2012;10:2471-2488.

34. Ortiz S, Pérez-Merino P, Durán S, et al. Full OCT anterior segment biometry: an application in cataract surgery. Biomed Opt Express. 2013;4:387-396.

35. Maeda N, Klyce SD, Smolek MK, Thompson HW. Automated keratoconus screening with corneal topography analysis. Invest Ophthalmol Vis Sci. 1994;35:2749-2757.

36. Rabinowitz YS, McDonnell PJ. Computer-assisted corneal topography in keratoconus. Refract Corneal Surg. 1989;5: 400-408.

37. Maguire LJ, Bourne WM. Corneal topography of early keratoconus. Am J Ophthalmol. 1989;108:107-112.

38. Tomidokoro A, Oshika T, Amano S, Higaki S, Maeda N, Miyata $\mathrm{K}$. Changes in anterior and posterior corneal curvatures in keratoconus. Ophthalmology. 2000;107:1328-1332.

39. Colin J, Cochener B, Savary G, Malet F, Holmes-Higgin D. INTACS inserts for treating keratoconus: one-year results. Ophthalmology. 2001;108:1409-1414.

40. Söğütlü E, Piñero DP, Kubaloglu A, Alio JL, Cinar Y. Elevation changes of central posterior corneal surface after intracorneal ring segment implantation in keratoconus. Cornea. 2012;31: 387-395.

41. Piñero DP, Alio JL, El Kady B, et al. Refractive and aberrometric outcomes of intracorneal ring segments for keratoconus: mechanical versus femtosecond-assisted procedures. Ophthalmology. 2009;116:1675-1687.

42. Chen M, Yoon G. Posterior corneal aberrations and their compensations effects on anterior corneal aberrations in keratoconic eyes. Invest Ophthalmol Vis Sci. 2008;49:56455652 . 